Ann. Biol. anim. Bioch. Biophys., I969, 9 (2), 233-250.

\title{
VARIATIONS SAISONNIÈRES DU COMPORTEMENT D'CESTRUS ET DES ACTIVITÉS OVARIENNE ET HYPOPHYSAIRE CHEZ LES OVINS
}

\author{
J. THIMONIER, P. MAULÉON \\ avec la collaboration technique de Jacqueline BÉzARD, Marie-Madeleine DE Reviers \\ et C. CORNu \\ Laboratoire de Physiologie de la Reproduction, \\ Centre de Recherches vétérinaires et zootechniques, 37 - Nouzilly \\ Instiut national de la Recherche agronomique
}

\section{SOMMAIRE}

Le comportement d'œstrus cyclique et l'activité ovarienne ont été étudiés chez les mêmes brebis de races Ile-de-France et Préalpes pendant deux années consécutives. Il existe des différences raciales dans la durée de l'anœestrus saisonnier. Les brebis de race Préalpes ont un anœestrus de I 44 jours, et celles de race Ile-de-France, de I 79 jours.

Des différences individuelles ont été mises en évidence. Ainsi, certaines brebis de race Préalpes n'ont pas d'anœestrus, d'autres au contraire ont un anœstrus très long. Toutes les brebis de race Ile-deFrance ont une période d'anœstrus mais la durée de celle-ci peut varier du simple au double.

Une reprise de l'activité ovarienne, contrôlée par coelioscopie, est observée chez les deux races fin avril-début mai.

Dans les 2 races, l'activité ovarienne reprend au mois de juin, en jours longs croissants, contrairement à ce que les études antérieures sur le photopériodisme suggéraient.

Chez les brebis de race Ile-de-France, pendant la période d'anostrus, les teneurs hypophysaires en FSH et LH sont 2 fois plus faibles que celles trouvées dans les hypophyses pendant la saison sexuelle.

\section{INTRODUCTION}

L'existence de variations saisonnières du comportement d'œestrus et de l'activité ovarienne a souvent été rapportée (voir revue bibliographique : OrTAVANT, MAULÉoN, ThibaulT, r964). Cependant, selon les races, on constate des différences dans la valeur des paramètres qui définissent les caractéristiques de la saison sexuelle (RouX, I936 ; HAFEZ, I952 ; HunTER, I964 ; KARDYMowICZ, I964). En outre, il 
existe des variations individuelles importantes, au sein d'une même race (MIL, ER, I962 ; KARDYMOWICZ, I964), variations dont la connaissance peut permettre éventuellement une sélection génétique d'animaux à saison sexuelle plus ou moins longue.

L'activité sexuelle saisonnière est contrôlée essentiellement par les variations de la photopériode qui entraînent des modifications de 1'activité hypophysaire ('THIBAUL,T et al., I966). Cependant, les résultats concernant ce dernier point sont contradictoires (COLE et MILIEER, I935 ; WARWICK, I946 ; KAMMLADE et al., I952 ; LAMOND, RADFORD, WALIACE, I959 ; HutChINSON et RoBER'TSON, I96o ; REEVES, I967).

Nos connaissances sur les races françaises sont peu précises. Aussi avons-nous cherché à suivre individuellement les activités œestrale et ovarienne de brebis appartenant à deux races, Ile-de-France et Préalpes, pendant plusieurs années consécutives et étudié les teneurs hypophysaires en FSH et $\mathrm{LH}$ à différents moments de l'année.

\section{MATÉRIEI, ET MÉTHODES}

\section{Animaux}

Dix-sept animaux de chacune des deux races, Ile-de-France et Préalpes, ont été l'objet de cette étude de 1963 à 1966 . Les caractéristiques de ces races et leur origine ont été définies par MaULÉON et ROUGEOT (I962).

Les observations ont été faites à Jouy-en-Josas (latitude $48^{\circ} 30^{\prime} \mathrm{N}$ ).

Les brebis Ile-de-France et Préalpes, nées au printemps 1962 , n'ont pas été saillies pendant toute la durée des observations.

A l'âge de deux ans et demi, en octobre 1964 , les brebis Ile-de-France pesaient en moyenne $65,5 \mathrm{~kg}$ et les brebis Préalpes $60 \mathrm{~kg}$.

Les animaux des deux races ont été gardés sous un même abri et ont reçu la même alimentation : fourrages verts en été ; collets de betteraves, ensilage et foin pendant l'hiver. Une ration quotidienne de $200 \mathrm{~g}$ de céréales (blé, orge, avoine) a été distribuée.

La détection de l'œstrus a été effectuée une fois par jour selon la méthode précédemment décrite par MAULÉON et DAUZIER (1965). Le critère du comportement d'œestrus est constitué par l'immobilité de la brebis pendant le chevauchement du mâle.

\section{Contrôle de l'activité ovarienne}

La laparotomie ne permet généralement pas les observations ovariennes répétées de nombreuses fois sur le même animal car des adhérences se produisent souvent entre épiploon et ovaire gênant le contrôle de l'activité ovarienne et pouvant perturber la fonction ovarienne.

La laparoscopie fréquemment utilisée en gynécologie humaine (PALMER, I964) permet d'envisager un nombre plus important de contrôles ovariens sur un même animal.

a) Appareil.

Nous avons utilisé le laparoscope « Universal ( $\left.{ }^{1}\right)$ ". Une optique à grand champ facilite l'orientation dans l'abdomen. Un petit projecteur extérieur, d'une puissance de Io watts fournit une lumière plus forte qu'une lampe distale classique.

L'endoscope est introduit dans la cavité générale grâce à un trocard rond de I I $\mathrm{mm}$ de diamètre. Une pince à biopsie rigide sert à découvrir et retourner les ovaires de façon à les regarder sur toutes les faces.

\section{b) Mode opératoire.}

L'examen étant rapide, tous les animaux ont été opérés sous anesthésie générale légère (Nembutal-pentothal). La brebis est mise en décubitus dorsal, et deux incisions cutanées de $1 \mathrm{~cm}$ sont faites symétriquement par rapport à la ligne médiane ventrale, à environ $\mathrm{I} 5 \mathrm{~cm}$ en avant de la mamelle.

Après avoir traversé la paroi musculaire à l'aide du trocard, l'endoscope est introduit par la chemise de celui-ci. Des insufflations d'air permettent de rejeter la masse intestinale enveloppée par

(1) Storz : Tuttlingen, Hermanstrasse $\mathrm{r}_{4}$, Süddeutschland. 
l'épiploon. Par la seconde ouverture, la pince à biopsie est mise en place puis l'animal est basculé, tête vers le bas, pour dégager encore plus le tractus génital de la masse intestinale. Les ovaires souvent cachés sous le ligament large sont facilement dégagés avec la pince à biopsie.

Après examen, I 000000 unités de pénicilline sont introduites dans la cavité générale par le trocard. Deux agrafes suffisent pour refermer les incisions cutanées ; la suture de la paroi musculaire est inutile.

Si l'anesthésie des animaux, leur mise en place sur la table d'opération et leur enlèvement sont réalisés par deux aides, il est possible d'examiner en moyenne $\mathrm{I} 2$ brebis en $\mathrm{I}$ heure.

En évitant de léser les ovaires et le tractus génital avec la pince à biopsie, et de provoquer des hémorragies internes même légères, 40 endoscopies peuvent être faites sur un même animal.

Nous avons utilisé uniquement l'anesthésie générale pour nos observations. L'emploi des tranquillisants avec anesthésie locale est cependant suffisant pour effectuer cette courte intervention chirurgicale.

\section{c) Fréquence des observations.}

Nous avons supposé que tout œstrus régulier était accompagné d'une ovulation. Le contrôle ovarien a été réalisé toutes les fois que les brebis ne venaient pas normalement en œstrus pendant la saison sexuelle et pendant toute la période d'ancestrus : tous les 17 jours pour les brebis ayant des ovulations silencieuses, tous les 34 jours pour les brebis sans activité ovarienne et ceci pendant deux années consécutives.

Suivant les brebis, 30 à 40 contrôles ovariens par animal ont été effectués pendant cette période de 2 ans.

\section{Dosages des activités hypophysaires en FSH et LH}

L'étude a été faite sur une seule race. 93 brebis de race Ile-de-France ayant les mêmes caractéristiques de reproduction (époque de lutte, saison sexuelle) que celles observées par endoscopie, ont été abattues.

Les abattages ont été effectués mensuellement le matin entre 9 et Io heures.

Lorsque les brebis étaient cycliques, les prélèvements du matériel hypophysaire ont été faits vers le $12^{\mathrm{e}}$ jour du cycle, moment où la charge hypophysaire en FSH et LH est maximum (ROBERTson et HuTCHINSON, I 6 62). Chez les brebis ayant des ovulations silencieuses, le $12^{\mathrm{e}}$ jour du cycle a été déterminé d'après l'examen macroscopique des corps jaunes par endoscopie.

Les hypophyses des brebis abattues ont été conservées dans l'acétone à $-15^{\circ} \mathrm{C}$ et broyées.

Les activités FSH et LH ont été dosées sur des rattes Wistar (souche ${ }_{3} 3$ du C. N. R. Z.) respectivevement par la méthode de SteElman et Polhey (1953) et par la technique de Parlow modifiée par Pelletier (I963).

\section{Analyse statistique des résultats}

La détermination de la signification statistique a été effectuée soit par le test $t$ de Student, soit par le test du $\chi^{2}$.

\section{RÉSULTATS}

\section{A. - Comportement d'estrus et activité ovarienne}

Connaissant la durée du cycle ostrien propre à une brebis, il est possible d'estimer le nombre théorique d'cestrus qu'elle pourrait avoir en un an s'il n'existait pas de période d'ancestrus.

A tout œestrus est associé une possibilité d'ovulation. Le nombre annuel théorique d'œestrus ou de possibilités d'ovulation varie selon les animaux de Ig à 23 pour les brebis des deux races.

On peut alors exprimer le nombre d'œstrus et d'ovulations réellement observés en pourcentage du nombre théoriquement possible (tabl. I, fig. I, 2, 3). 
TABLEAU I

Comportement d'cestrus et activité ovarienne chez les brebis de races Préalpes et lle-de-France pendant la période du 1-11-1963 au 31-10-1965

\begin{tabular}{|c|c|c|c|c|c|c|}
\hline Race & $\begin{array}{c}\text { Nombre } \\
\text { de Brebis (1) }\end{array}$ & $\begin{array}{c}\text { Nombre théorique } \\
\text { d'œestrus ou } \\
\text { de possibilités } \\
\text { d'ovulation }\end{array}$ & $\begin{array}{l}\text { Nombre } \\
\text { d'œestrus } \\
\text { observés }\end{array}$ & $\begin{array}{l}\text { P. } 100 \\
\text { d'œstrus } \\
\text { observés }\end{array}$ & $\begin{array}{c}\text { Nombre } \\
\text { d'ovulations } \\
\text { observées }\end{array}$ & $\begin{array}{c}\text { P. } 100 \\
\text { d'ovulations } \\
\text { observées }\end{array}$ \\
\hline $\begin{array}{l}\text { Préalpes .... } \\
\text { Ile de France. }\end{array}$ & $\begin{array}{r}9 \\
14\end{array}$ & $\begin{array}{l}372 \\
606\end{array}$ & $\begin{array}{l}238 \\
289\end{array}$ & $\begin{array}{l}64,0 \\
47,7^{* *}\end{array}$ & $\begin{array}{l}328 \\
425\end{array}$ & $\begin{array}{l}88,2 \\
70,1^{* *}\end{array}$ \\
\hline
\end{tabular}

(1) Uniquement les brebis ayant vécu pendant toute la durée des observations (1-11-1963 au 31-10-1965).

** Différences entre les 2 races : hautement significatives.
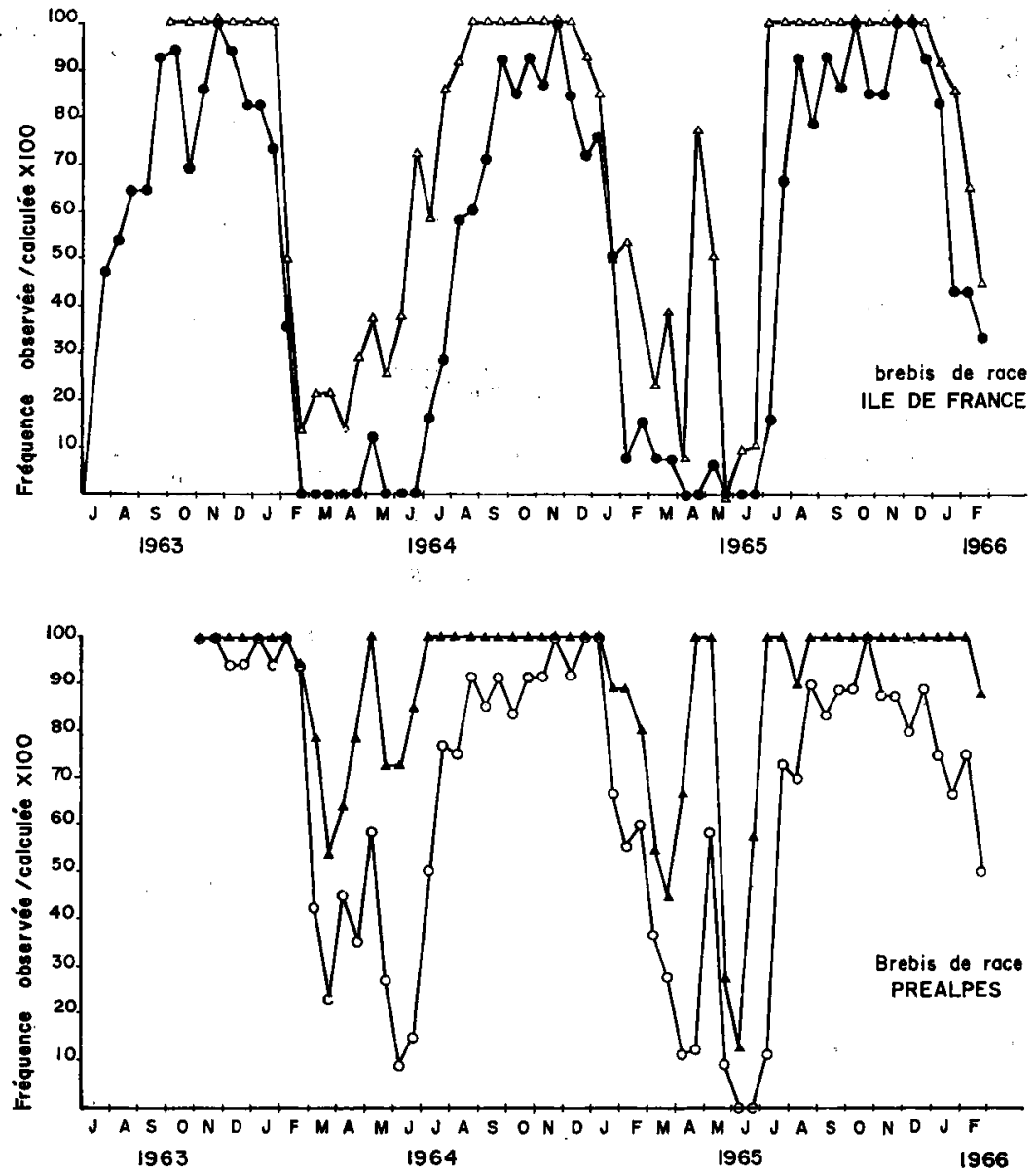

FIG. I. - Variations annuelles de la fréquence observée

du comportement d'estrus (0-O) et de l'ovulation $(\Delta-\Delta)$ dans des groupes de brebis des races Ile-de-France et Préalpes 


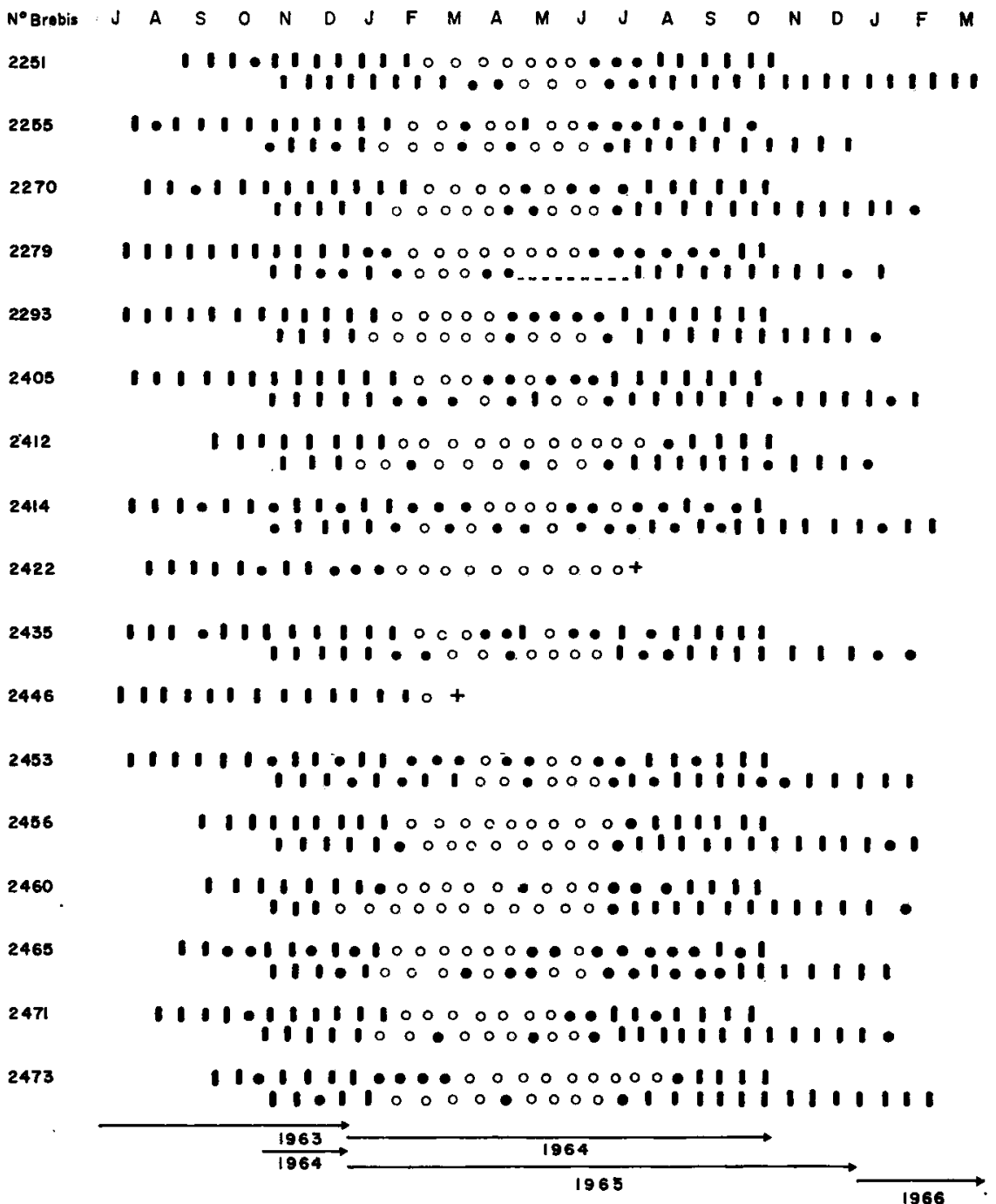

FIG. 2. - Répartition des astrus et des ovulations au cours de l'année pour chacune des brebis de race Ile-de-France

œestrus

- ovulation silencieuse

- cestrus et ovulation non observés

+ brebis morte

- brebis n'ayant pu être observée 


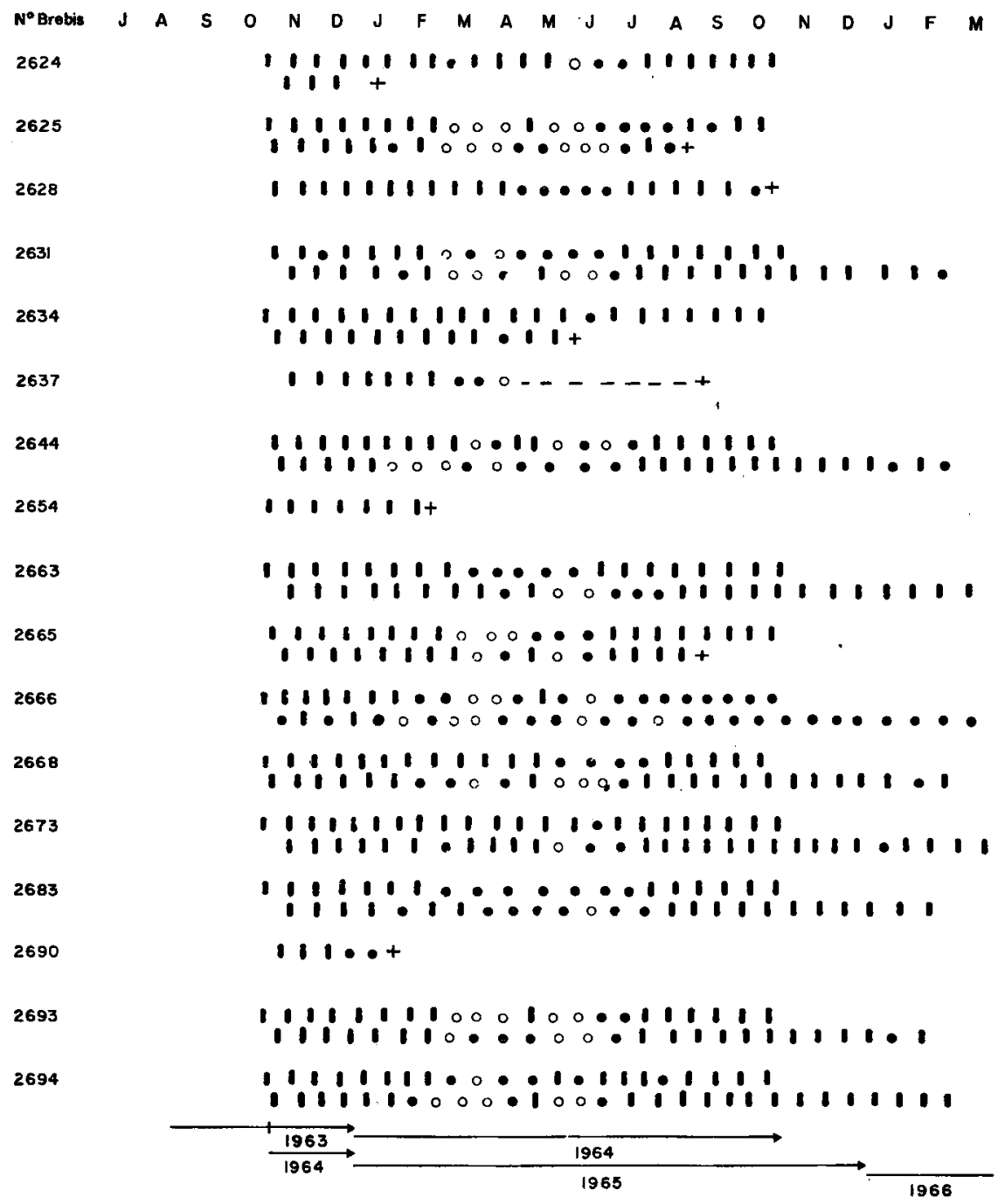

Fig. 3. - Répartition des oestrus et des ovulations au cours de l'année pour chacune des brebis de race Préalpes

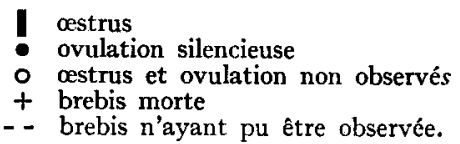




\section{Caractéristiques communes aux deux races.}

Les brebis Ile-de-France et Préalpes extériorisent un comportement d'œsrus dans respectivement 47,7 p. Ioo et 64,0 p. Ioo des cas (tabl. I). Il existe donc des arrêts de 1'activité cyclique du comportement d'œstrus. Ces arrêts sont plus fréquents certains mois de l'année définissant une période d'anœstrus (fig. I).

Vers le milieu de cette période d'anœstrus, quelques brebis des deux races présentent des œstrus isolés accompagnés d'ovulation, sans reprise cyclique du comportement d'œstrus. Ce phénomène est plus fréquent pour les brebis de race Préalpes que pour celles de race Ile-de-France (fig. I, 2 et 3 ). L'ovulation a été observée dans 70 à 88 p. Ioo des possibilités d'ovulation chez les brebis de race Ile-de-France et Préalpes (tabl. I). Il se produit donc moins souvent d'arrêts de l'activité ovarienne que de suppression du comportement d'œstrus. Des ovulations silencieuses se produisent dans 22,4 et 24,3 p. Ioo des cas chez les brebis de races Ile-de-France et Préalpes. Une telle dissociation cestrus-ovulation existe tout au long de l'année avec plus ou moins d'intensité.

Après 1'arrêt du comportement cyclique d'œestrus, 35 à 40 p. Ioo seulement des animaux, quelles que soient l'année et la race, ont des ovulations silencieuses. Par contre, tous les animaux présentent de telles ovulations avant la reprise du comportement cyclique (tabl. 2).

\section{TABLEAU 2}

Anoestrus, comportement d'cestrus cyclique et ovulations silencieuses au cours de l'année chez les brebis des races Préalpes et Ile-de-France

(en p. Ioo des brebis présentes pendant les périodes considérées)

\begin{tabular}{|c|c|c|c|c|}
\hline Race & \multicolumn{2}{|c|}{ Préalpes } & \multicolumn{2}{|c|}{ Ile de France } \\
\hline Période & $\begin{array}{r}1-11-63 \\
31-10-64\end{array}$ & $\begin{array}{r}1-11-64 \\
31-10-65\end{array}$ & $\begin{array}{r}1-11-63 \\
31-10-64\end{array}$ & $\begin{array}{r}1-11-64 \\
31-1064\end{array}$ \\
\hline $\begin{array}{c}\text { Comportement } d \text { 'ostrus cyclique toute } \\
\text { l'année } \ldots \ldots \ldots \ldots \ldots \ldots \ldots \ldots \ldots \ldots \ldots\end{array}$ & 13,3 & 0 & 0 & 0 \\
\hline Ovulations toute l'année $\ldots \ldots \ldots \ldots \ldots$ & 40,0 & 0 & 0 & 0 \\
\hline Ancestrus complet $\ldots \ldots \ldots \ldots \ldots \ldots$ & 60,0 & 100,0 & 100,0 & 100,0 \\
\hline $\begin{array}{l}\text { Ovulations silencieuses après la fin de la } \\
\text { saison sexuelle.................... }\end{array}$ & 40,0 & 36,4 & 35,3 & 40,0 \\
\hline $\begin{array}{l}\text { Ovulations silencieuses avant le début de } \\
\text { la saison sexuelle.................. }\end{array}$ & 100,0 & 100,0 & 100,0 & 92,9 \\
\hline $\begin{array}{l}\text { Anœestrus complet interrompu par des ovu- } \\
\text { lations silencieuses. . . . . . . . . . . }\end{array}$ & 62,5 & 72,7 & 53,3 & 78,6 \\
\hline $\begin{array}{c}\text { Ovulations silencieuses pendant la saison } \\
\text { sexuelle } \ldots \ldots \ldots \ldots \ldots \ldots \ldots \ldots \ldots \ldots\end{array}$ & 57,1 & 55,6 & 53,3 & 46,7 \\
\hline
\end{tabular}




\section{Variations entre races.}

La durée moyenne de l'anœstrus saisonnier a été calculée en faisant la moyenne des durées individuelles de l'ancestrus de chaque brebis de chacune des 2 races. En considérant les deux années, la durée moyenne de l'anœestrus des brebis de race Ile-de-France est de 179 jours, c'est-à-dire beaucoup plus longue que celle des brebis de race Préalpes qui est de II4 jours (tab1. 3). Cette différence de durée de l'anœstrus explique que pour les brebis de race Ile-de-France, 48 p. Ioo seulement des chaleurs possibles aient été exprimées contre 64 p. Ioo pour les brebis de race Préalpes.

La date moyenne du premier œestrus de la saison sexuelle est le 27 juillet pour les animaux de race Préalpes. Chez les brebis de race Ile-de-France, 2 années sur 3, elle se situe vers le I5 août. En I965, cependant, cette date moyenne est légèrement antérieure à celle calculée pour les brebis de race Préalpes (tabl. 3). Dans 2 cas sur 3 la saison sexuelle des brebis de race Préalpes débute donc au moins I 8 jours plus tôt que celle des brebis de race Ile-de-France.

La date moyenne du dernier œstrus de la saison sexuelle varie selon les années du 2 I février au 4 mars pour les brebis de race Préalpes et du I 7 au 25 janvier pour les brebis de race Ile-de-France. L'ancestrus saisonnier débute donc, au moins, un mois plus tôt chez les brebis de race Ile-de-France, que chez les brebis de race Préalpes.

La comparaison des dates auxquelles 50 p. Ioo des femelles de chacune des deux races ont leur premier œstrus et des dates auxquelles $50 \mathrm{p}$. Ioo ont le dernier œestrus de la saison sexuelle, confirme que l'anœstrus saisonnier est beaucoup plus long chez les brebis Ile-de-France que chez les brebis de race Préalpes (tabl. 3).

Enfin, pendant la période d'ancestrus, on constate un plus grand nombre d'ovulations silencieuses chez les brebis de race Préalpes que chez les brebis de race Ile-deFrance (fig. I).

\section{Variations annuelles.}

Elles sont pratiquement inexistantes pour les animaux Ile-de-France. En I965, cependant, nous avons observé que la date moyenne de début de la saison sexuelle était avancée de 20 jours (tab1. 3 ).

En race Préalpes, les chaleurs ont été plus fréquentes au cours de l'année Ig63I964 qu'en I964-I965:59,0 p. Ioo des œstrus théoriquement possibles ont été extériorisés pendant cette dernière période, au lieu de $70,9 \mathrm{p}$. roo pendant la période précédente (tabl. 4). Une variation dans le même sens existe pour les moments d'ovulation. Mais les dates moyennes des premier et dernier œestrus de la saison sexuelle sont identiques pour les deux périodes considérées (tab1. 3).

\section{Variations individuelles.}

Une même brebis peut présenter différents états physiologiques au cours de l'année.

- comportement d'œestrus cyclique ;

- anœstrus mais avec activité ovarienne cyclique ;

- ancstrus, sans ovulation que nous appellerons ancstrus complet. 
VARIATIONS SAISONNIÈRES

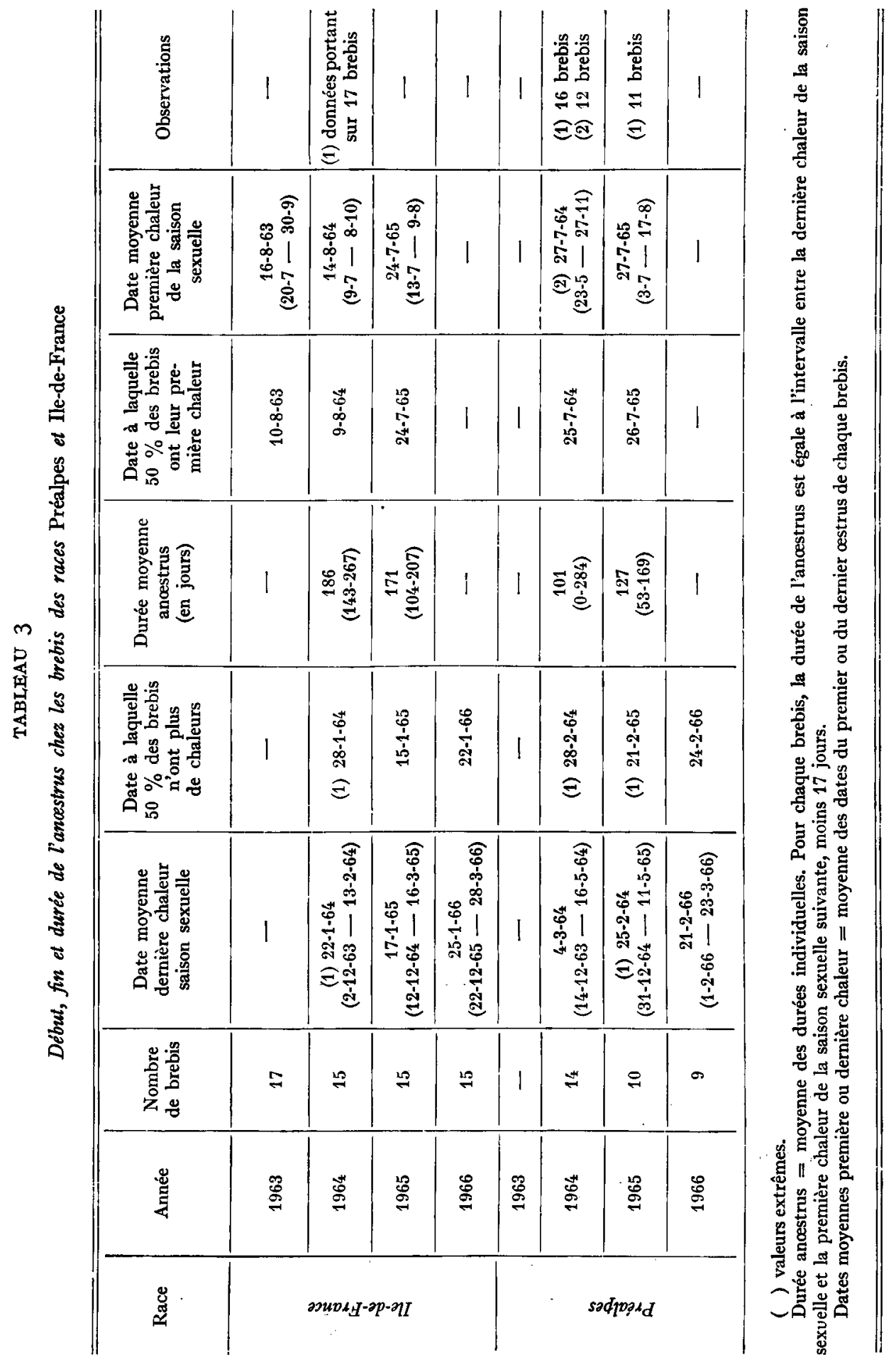


La présence d'un anœstrus complet généralisé est une caractéristique des brebis de race Ile-de-France. Mais la moitié ou les trois quarts de ces animaux, suivant les années, ont leur anœstrus interrompu par des ovulations ou des chaleurs fin avril, début mai (tabl. 2). A l'exception de deux brebis (225I et 2456), toutes ont présenté ce phénomène au moins une fois au cours des deux années (fig. 2), mais des variations du simple au double dans la durée de la saison d'anœstrus existent entre brebis.

Les variations individuelles en race Préalpes sont encore plus importantes. Certaines brebis n'ont jamais d'anœstrus complet (fig. 3), d'autres au contraire ont un long anœstrus qui est fréquemment coupé par des ovulations silencieuses.

\section{TABLEAU 4}

Variations annuelles du comportement d'astrus et de l'activité ovarienne chez les brebis des races Préalpes et Ile-de-France

\begin{tabular}{|c|c|c|c|c|c|c|c|}
\hline Race & $\begin{array}{l}\text { Période } \\
\text { considérée }\end{array}$ & $\begin{array}{l}\text { Nombre } \\
\text { de Brebis } \\
\text { (1) }\end{array}$ & $\begin{array}{l}\text { Nbre théorique } \\
\text { d'œestrus ou } \\
\text { de possibilités } \\
\text { d'ovulation }\end{array}$ & $\begin{array}{l}\text { Nombre } \\
\text { d'œstrus } \\
\text { observés }\end{array}$ & $\begin{array}{l}\text { P. } 100 \\
\text { d'œstrus } \\
\text { observés }\end{array}$ & $\begin{array}{c}\text { Nombre } \\
\text { d'ovulations } \\
\text { observées }\end{array}$ & $\begin{array}{c}\text { P. } 100 \\
\text { d'ovulations } \\
\text { observées }\end{array}$ \\
\hline \multirow{2}{*}{ 胥 } & $\begin{array}{r}1-11-63 \\
31-10-64\end{array}$ & 14 & 296 & 210 & 70,9 & 272 & 91,9 \\
\hline & $\begin{array}{r}1-11-64 \\
31-10-65\end{array}$ & 9 & 183 & 108 & $59,0^{* *}$ & 154 & $84,2^{*}$ \\
\hline \multirow{2}{*}{  } & $\begin{array}{r}1-11-63 \\
31-10-64\end{array}$ & 15 & 330 & 150 & 45,5 & 232 & 70,3 \\
\hline & $\begin{array}{r}1-11-64 \\
31-10-65\end{array}$ & 14 & 298 & 145 & 48,7 & 207 & 69,5 \\
\hline
\end{tabular}

(1) Uniquement les brebis ayant survécu pendant la période considérée.

Différences entre les deux périodes considérées $\begin{cases}\text { significative } & * \mathrm{P}<0,05 \\ \text { hautement significative } & * * \\ \mathrm{P} & <0,01\end{cases}$

\section{B. - Activité hypophysaire}

L'éturde des teneurs hypophysaires en FSH et LH a été faite tout au long de l'année, en tenant compte de l'état physiologique des animaux.

L'effet de l'anesthésie a été contrôlé sur les brebis ayant un comportement d'œstrus cyclique. Aucune influence significative n'étant apparue, on peut donc considérer que les brebis en anœstrus ont des teneurs hypophysaires en FSH et LH voisines de celles qu'elles auraient eu si cette intervention chirurgicale n'avait pas eu lieu.

\section{Teneurs hypophysaires en hormones gonadotropes.}

Le poids des hypophyses subit une légère diminution vers le printemps (tab1. 5), et le poids des hypophyses des brebis en ancestrus est plus faible que celui des hypo- 
physes des animaux ayant une activité cyclique ovarienne (tabl. 6). Ces variations ne sont cependant pas significatives. La comparaison entre les différents lots pourra donc se faire sur les concentrations en FSH et LH des hypophyses.

TABLEAU 5

Variation saisonnière du poids des hypophyses

\begin{tabular}{c|c|c|c|c}
\hline Période & Déc.-Janv.-Fév. & Mars-Avril-Mai & Juin-Juil.-Août & Sept.-Oct.-Nov. \\
\hline $\begin{array}{c}\text { Poids des hypophyses } \\
\text { (g.) }\end{array}$ & $0,84 \frac{ \pm 0,030}{(32)}$ & $0,77 \frac{ \pm 0,044}{(18)}$ & $0,79 \frac{ \pm 0,024}{(40)}$ & $0,86 \frac{ \pm 0,069}{(12)}$ \\
\hline
\end{tabular}

( ) Nombre de brebis.

\section{TABLEAU 6}

Variations du poids des hypophyses

en fonction de l'état physiologique

\begin{tabular}{c|c|c|c}
\hline \hline État physiologique & $\begin{array}{c}\text { Brebis ayant } \\
\text { un comportement } \\
\text { d'oestrus cyclique }\end{array}$ & $\begin{array}{c}\text { Brebis } \\
\text { ayant des ovulations } \\
\text { silencieuses }\end{array}$ & $\begin{array}{c}\text { Brebis } \\
\text { en anœstrus complet }\end{array}$ \\
\hline $\begin{array}{c}\text { Poids des hypophyses } \\
(\mathrm{g} .)\end{array}$ & $0,80 \frac{ \pm 0,036}{(47)}$ & $0,83 \frac{ \pm 0,030}{(32)}$ & $0,76 \frac{ \pm^{(23)} 0,071}{(23)}$ \\
\hline
\end{tabular}

( ) Nombre de brebis

La teneur moyenne en FSH pendant les mois de mars, avril et mai varie entre 2,25 et 3,93 $\mu \mathrm{g}$ équivalent $\mathrm{FSH}-\mathrm{NIH}-\mathrm{S}_{1} / \mathrm{mg}$ de poudre hypophysaire. Elle est deux fois plus faible que la concentration notée pendant les mois d'octobre, novembre et décembre (tabl. 7).

Une diminution de la teneur hypophysaire en FSH par rapport à celle obtenue pendant la saison sexuelle apparaît au mois de janvier.

En juin, juillet et août, on observe une concentration intermédiaire en FSH dans les hypophyses.

Le manque de sensibilité du dosage biologique de l'activité FSH n'a pas permis de déterminer la concentration en cette hormone dans les pools d'hypophyses provenant de trop petits nombres d'animaux (lot III). Néanmoins, la teneur dans les hypophyses des animaux à ovulations silencieuses au mois de février est plus proche de celle des hypophyses des animaux en saison sexuelle, que de celles des brebis en ancestrus complet. Toutefois, cette conclusion n'a pu être confirmée au mois de juillet (tabl. 5). 
TABLEAU 7

Variation saisonnière de la teneur en FSH des hypophyses de brebis de race Ile-de-France

(en $\mu$ g équivalent $\mathrm{FSH} / \mathrm{NIH} / \mathrm{S}_{1}$ par mg de poudre hypophysaire)

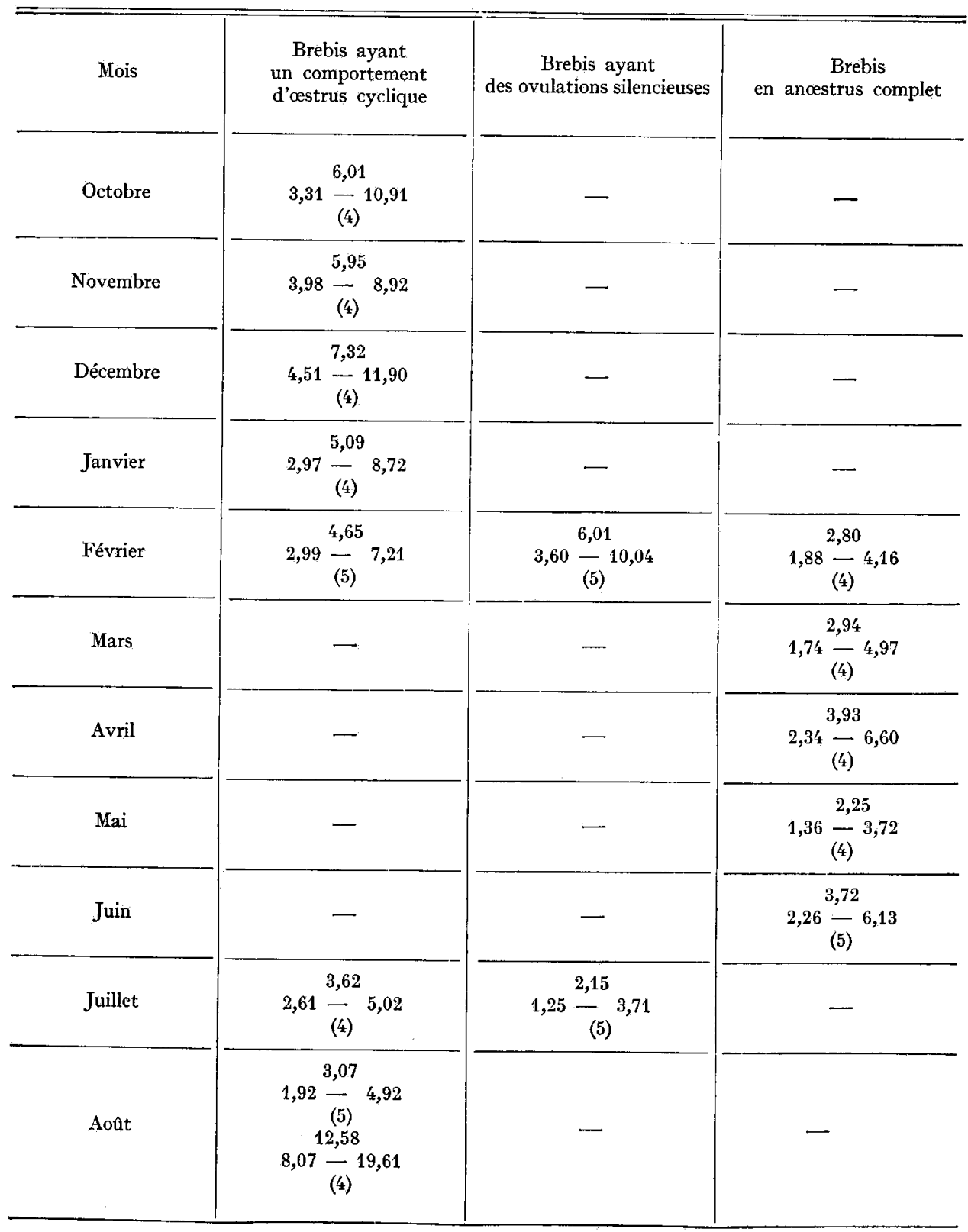

( ) Nombre de brebis utilisées. 
TABLEAU 8

Variation saisonnière de la teneur en $L H$ des hypophyses de brebis de race Ile-de-France (en $\mu \mathrm{g}$ équivalent $\mathrm{LH}-\mathrm{NIH} / \mathrm{S}_{\mathbf{1}}$ par $\mathrm{mg}$ de poudre hypophysaire )

\begin{tabular}{|c|c|c|c|}
\hline Mois & $\begin{array}{l}\text { Brebis ayant } \\
\text { un comportement } \\
\text { d'oestrus cyclique }\end{array}$ & $\begin{array}{c}\text { Brebis ayant } \\
\text { des ovulations silencieuses }\end{array}$ & $\begin{array}{c}\text { Brebis } \\
\text { en anœstrus complet }\end{array}$ \\
\hline Octobre & $\begin{array}{c}13,13 \\
9,29-18,55 \\
(3) \\
20,14 \\
12,58 \frac{-}{(4)} 32,24\end{array}$ & - & - \\
\hline Novembre & $16,94 \frac{25,28}{(4)} 37,74$ & - & - \\
\hline Décembre & $12,24 \frac{17,98}{(4)} 26,42$ & - & - \\
\hline Janvier & $5,20 \frac{8,28}{(4)} 13,10$ & $\begin{array}{c}10,95 \\
7,33 \underset{(1)}{\longrightarrow} 16,37 \\
4,82 \frac{7}{(6)} 12,85\end{array}$ & - \\
\hline Février & $6,71 \frac{11,29}{(5)} 19,00$ & $3,79 \frac{5,25}{(1)} 7,27$ & $5,70 \frac{8,25}{(4)}^{\frac{84}{4}} 14,42$ \\
\hline Mars & - & - & $6,83 \frac{11,00}{(4)} 17,68$ \\
\hline Avril & 一 & $5,89 \frac{8,12}{(2)} 11,20$ & $8,53 \frac{12,47}{(4)} 18,50$ \\
\hline Mai & - & $4,83 \frac{7,30}{(2)} 11,03$ & $\begin{array}{l}3,27 \frac{6,01}{(1)} 11,05 \\
3,56 \frac{5,48}{(4)} 8,41\end{array}$ \\
\hline Juin & $7,15 \frac{10,90}{(4)} 16,60$ & 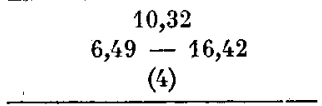 & $\begin{array}{c}15,57 \\
10,70-22,65 \\
(5)\end{array}$ \\
\hline Juillet & $\begin{array}{c}15,60 \\
10,02 \frac{-}{(4)} 24,25 \\
13,35 \\
6,49 \frac{-16,42}{(1)}\end{array}$ & $\begin{array}{c}8,20 \\
5,76 \frac{(4)}{(4)} 11,61 \\
21,09 \\
13,12 \frac{-}{(5)} 33,92\end{array}$ & 一 \\
\hline Août & $\begin{array}{c}13,22 \\
9,09 \frac{(4)}{-} 19,21 \\
10,17 \frac{13,97}{(5)} 19,20\end{array}$ & 一 & - \\
\hline
\end{tabular}

Valeurs des $\lambda$ (Index de précision) : entre 0,177 et 0,276. ( ) Nombre de brebis utilisées. 
$\mathrm{La}_{\mathrm{a}}$ teneur en hormone lutéinisante $(\mathrm{LH})$ est comprise entre 5,48 et $\mathrm{I} 2,47 \mu \mathrm{g}$ équivalent $\mathrm{L} / \mathrm{H}-\mathrm{NIH} \mathrm{S}_{\mathbf{1}} / \mathrm{mg}$ de poudre hypophysaire, pendant les mois de mars, avril et mai. Cette concentration est environ deux fois plus faible que celle trouvée dans les hypophyses pendant les mois d'octobre, novembre et décembre (tabl. 8).

Pendant les mois de janvier, juin, juillet et août, les teneurs hypophysaires en $\mathrm{L}_{1} \mathrm{H}$ sont intermédiaires.

Lorsque les ovulations silencieuses se produisent pendant la saison d'anœstrus, les teneurs hypophysaires en $L_{1} H$ sont plus faibles chez ces animaux que celles observées chez les animaux en anœstrus complet.

2. Autres glandes endocrines (tabl. 9).

Le poids des surrénales ne subit aucune variation saisonnière. Le poids des thyroìdes est minimum en décembre, janvier et février, et maximum en juin, juillet août. Les glandes pinéales des brebis sont plus légères pendant les mois de mars, avril, mai.

\section{TABLEAU 9}

Variations saisonnières du poids des thyroìdes, surrénales, pinéales chez les brebis de race Ile-de-France

\begin{tabular}{|c|c|c|c|c|}
\hline Période de l'année & $\underset{1}{\text { Déc.-Janv.-Fév. }}$ & $\begin{array}{l}\text { Mars-Avril-Mai } \\
2\end{array}$ & $\underset{3}{\text { Juin-Juil.-Août }}$ & $\underset{4}{\text { Sept.-Oct.-Nov. }}$ \\
\hline $\begin{array}{l}\text { Poids des glandes thyroïdes } \\
\text { (g) }\end{array}$ & $3,00 \underset{(32)}{ \pm} 0,133$ & $3,41 \underset{(18)}{ \pm} 0,247$ & $4,11 \underset{(40)}{ \pm} 0,217$ & $3,74 \frac{ \pm}{(11)}^{0,374}$ \\
\hline $\begin{array}{l}\text { Poids des glandes surrénales } \\
\text { (g) }\end{array}$ & $2,94 \frac{ \pm}{(32)}^{0,065}$ & $2,76 \underset{(18)}{ \pm} 0,122$ & $2,81 \underset{(40)}{ \pm} 0,059$ & $\left.{ }_{(12)}^{2,89}\right)^{0,141}$ \\
\hline $\begin{array}{c}(\mathrm{g}) \\
\text { Poids des glandes pinéales }\end{array}$ & $0,115 \pm \frac{ \pm}{(32)} 0,0056$ & $0,095 \underset{\frac{ \pm}{(17)}}{0,0080} 0$ & $0,118 \pm \frac{ \pm 0,0055}{(40)}$ & $0,106 \pm \frac{ \pm}{(12)} 0,0066$ \\
\hline
\end{tabular}

( ) Nombre d'animaux.

Surrénales : aucune différence significative.

Pinéales : différence entre $\left\{\begin{array}{lll}1 & \text { et } 2 & \text { significative } P<0,05 \\ 1 & \text { et } 3\end{array}\right.$

Pinéales : difference entre $\left\{\begin{array}{l}2 \text { et } 3 \quad \text { significative } P<0,05 \\ 1 \text { a }\end{array}\right.$

Thyroides : différence entre $\left\{\begin{array}{l}1 \text { et } 3 \\ 2 \text { et } 3\end{array}\right.$ significative $\mathrm{P}<0,05 . \quad \mathrm{P}<0,01$

\section{DISCUSSION}

Il existe des variations saisonnières du comportement d'œestrus cyclique et de l'activité ovarienne. Au cours de l'année, apparaît une période d'anœestrus que l'on peut définir par son intensité et sa durée. 


\section{Intensité de l'ancestrus}

L'existence des ovulations silencieuses pendant la période d'anœstrus montre que l'anœstrus saisonnier n'a pas la même intensité pendant toute sa durée. La dissociation œestrus-ovulation a déjà été observée. Quelle est la raison de cette dissociation œstrus-ovulation ? RoBInson (I959) a montré que l'œstrus chez les Ovins nécessite un certain niveau œstrogénique après sensibilisation du système nerveux par la progestérone. En période d'anœstrus, le niveau œstrogénique peut être insuffisant, les hormones hypophysaires étant elles-mêmes insuffisantes.

Mais il existe également une variation saisonnière de sensibilité du système nerveux central (REARDON et ROBINSON, I96I).

La dissociation œstrus-ovulation pendant la saison sexuelle existe. Une insuffsance œstrogénique peut être également à l'origine de ces ovulations silencieuses. Dans certains cas, elles peuvent être attribuées à des œestrus très courts et non détectés.

\section{Durée de l'ancestrus et facteurs powvant la modifier}

Placées dans les mêmes conditions d'alimentation, de température, d'humidité et sous la même latitude, les animaux des deux races étudiées ont un anœstrus saisonnier qui diffère par sa durée. Les brebis de race Préalpes ont une période d'ancestrus beaucoup plus courte que les brebis de race Ile-de-France. Pour une même race, il existe des variations individuelles importantes : ainsi, certaines brebis de race Préalpes n'ont pas d'ancestrus saisonnier, d'autres, au contraire, ont une longue période de repos sexuel.

Ces différences raciales dans la durée de la saison d'anœstrus, sous une même latitude ont déjà été observées (Roux, I936; HAFEZ, I952 ; HuNTER, I964 ; KARDYMowicz, I964). Il en est de même pour les différences individuelles (MILLER, I962; KARDYMOWICZ, I964; LEES, I966).

Ce caractère durée de l'anœstrus est-il un caractère génétique ? Les expériences de HAFEZ (I952), montrent que la durée de l'anœestrus saisonnier est, chez des animaux résultant de croisements, intermédiaire entre celle des parents. Quelle est l'héritabilité de ce caractère ? Il n'existe pas à notre connaissance d'expérience satisfaisante sur ce sujet. L,es observations de LEES (I966) sur le comportement des mêmes brebis pendant deux années successives pourraient permettre de penser qu'une sélection est possible : une même brebis a un comportement reproductible chaque année. Notre étude confirme les observations de LEES : les brebis ayant eu un ancstrus complet court ou inexistant la première année, ont un anœstrus complet également court l'année suivante, particulièrement chez les animaux de race Préalpes. Mais WIILIAMs et al. (r956) constatent, au contraire, qu'il n'y a pas de tendance individuelle des brebis à revenir plus tôt ou plus tard en ostrus, à chaque saison. Dans ces conditions, le progrès génétique n'est pas certain.

Il est possible de déplacer le début et la fin de la saison sexuelle par une technique appropriée de conduite de troupeau. L'introduction de béliers vasectomisés ou entiers dans un troupeau, permet d'avancer la date de reprise du comportement d'œstrus (WATson et RADFORD, I960 ; EDGAR et BILKEY, I963 ; LYLE et HuNTER, r965). Le retrait et l'introduction périodiques des béliers en fin de saison sexuelle permettent de prolonger celle-ci (LAMOND, WELIS, MILLER, Ig63). 
L'alimentation et la température peuvent influencer le comportement d'œstrus cyclique (HAFEz, I952 ; SMITH, I966). Ces deux facteurs ne semblent pas avoir joué un rôle primordial sur la reprise ou la cessation du comportement d'œstrus cyclique pour nos animaux. Cependant, une température moyenne inférieure à la normale pendant les ro premiers jours du mois de juillet 1965 peut être à l'origine de la reprise plus rapide du comportement d'œstrus cyclique chez les brebis de race Ile-de-France.

Les traitements photopériodiques peuvent modifier le début de la saison sexuelle et la durée de celle-ci. (YEATES, I949; HART, I950 ; HAFEZ I95I ; MAULLÉON et RougEot, I962; ThWAITES, I965).

\section{Contrôle photopériodique du comportement d'estrus cyclique et des activités ovarienne et hypophysaire}

Pour la plupart des races, la période de saison sexuelle débute après le jour le plus long et s'arrête lorsque la croissance des jours a commencé (HAFEZ, I952). Dans le cas de nos deux races, le comportement d'œestrus cyclique cesse effectivement dans les mêmes conditions de photopériode que pour les autres races. Mais la reprise de l'activité ovarienne se manifeste dès la deuxième quinzaine du mois de juin, période qui correspond aux jours les plus longs.

Une reprise de l'activité ovarienne est observée également fin avril, début mai, alors que la durée de la photopériode claire augmente.

Les teneurs hypophysaires en $\mathrm{LH}$ et FSH sont faibles pendant toute la période d'anœstrus : cette diminution est une cause de l'ancestrus contrairement à l'opinion de WARWICK (1946). La corrélation entre les paramètres d'activité sexuelle et les taux hormonaux a été également mise en évidence chez le bélier par PELLETIER et ORTAVANT (I965).

Quoique non significative, une légère augmentation des teneurs hypophysaires en FSH et LH est observée au moment de la reprise d'activité ovarienne pendant le mois de juin. Ces augmentations se produisent en jours longs croissants contrairement à ce que les études de photopériodisme suggéraient jusqu'à présent.

Une explication est vraisemblablement à rechercher au niveau ovarien dans les variations de sécrétion des stéroïdes qui sensibilisent l'hypophyse aux ReleasingFactor. La présence fréquente des follicules pendant la période d'anœstrus peut faire penser qu'il existe en effet une sécrétion des hormones stéroïdiennes.

L'utilisation des techniques radio-immunologiques pour les dosages de FSH et LH ainsi que les dosages des stéroïdes dans le sang périphérique doivent nous permettre de mieux comprendre les mécanismes qui interviennent.

Reçu pour publication en novembre 1968.

\section{SUMMARY}

SEASONAL VARIATIONS IN GESTROUS BEHAVIOUR AND OVARIAN AND PITUITARY ACTIVITIES IN THE EWE

Estrous behaviour and ovarian activity have been studied over a two-year period in Ile-deFrance and Préalpes ewes. A celioscopic technique for monthly observation of ovaries allowed up to 4o ovarian controls on each ewe. The same animals were thus kept available for study for the two years of the experiment. 
The average seasonal anœstrous period was shorter in Préalpes (1 14 days) than in Ile-de-France (I79 days) ewes.

Considerable individual differences were pointed out. In the Préalpes, some ewes had regular œstrous cycles all the year round, whereas some had a prolonged anœstrous period. In the Ile-deFrance, all ewes typically showed a complete anostrous period, but the duration of the longest ancestrus was a multiple by 2 of that of the shortest.

Within the anœestrous periods, a short recovery of the ovarian activity was observed at the end of April or beginning of May.

The first ovarian cycles started in June under increasing day length, contrary to what previous literature on photoperiodism suggests.

In Ile-de-France ewes, the pituitary FSH and LH rates were lower during the ancstrous period than during the breeding season.

\section{RÉFÉRENCES BIBLIOGRAPHIQUES}

Cole H. H., Miller R. F., 3935. Changes in the reproductive organs of the ewe with some data bearing on their control. Amer. J. Anat., 57, 39-97.

EdGar D. G., BILKEY D. A., I963. Advancing the breeding season in ewes. Proc. Ruakura. Fmrs Cont. Week., 6-13.

HAFEZ E. S. E., I95I. Inhibitory action of artificial light on the sexual season of the ewe. Nature, Lond., 168, 336-337.

HAFEZ E. S. E., 1952. Studies on the breeding season and reproduction of the ewe. J. Agric. Sci., 42, I $89-265$.

HarT D. S., I950. Photoperiodicity in Sufolk sheep. J. Agric. Sci., 40, I43-149.

Hunter G. L., I964. The effects of season and mating on oestrus and fertility in the ewe. Proc. S. Afr. Soc. Anim. Prod., 3, 195-207.

Hutchinson J. S. M., Robertson H., ig6o. Effect of season on the follicle stimulating hormone and luteinzing hormone potency of sheep anterior pituitary glands. Nalure (Lond.), 188, 585-586.

Kammlade W. G., Welch J. A., Nalbandov A. V., Norton H. W., 3952. Pituitary activity of sheep in relation to the breeding season. J. Anim. Sci., 11, 646-655.

KaRDYMowICz M., I954. Comparison of breeding season, diøstrous cycle and œstrus period lengths in ewes of different breeds (en polonais) Roczn, Nauk, L. N., 88, 5.3-524.

LAMOND D. R., RADFORD H. M., WALlaCe A. L., 1959. Bioas say of sheep anterior pituitary glands Nature (Lond.), 183, 1597.

LAMOND D. R., Wells K. E., Miller S. J., ig63. Study of a breeding pro blem in Merino ewes in central Queensland. Aust. Vet. J., 39, 295-298.

LeES J. L., 1965. Variations in the time or onset of the breeding season in Clun ewes. J. Agric. Sci., 67, I 73-180.

LYLE A. D., HUNTER G. L., 1955. The effect on occurrence of œstrus in the ewe during spring of 6 and 14 day teasing periods prior to mating. Proc. S. Afr. Soc. Anim. Prod., 4, I 40-т 42.

Mauléon P., Dauzier L., 196.5. Variations de durée de l'anœstrus de lactation chez les brebis de race



MaulÉon P., Rougeot J., r962. Régulation des saisons sexuelles chez des brebis de races différentes au moyen de divers rythmes lumineux. Ann. Biol. anim. Bioch. Biophys., 2, 209-222.

MILLER W. W., 1962. Studies of ovarian activity and fertility in ewes and ewe lambs. Ph. D. Thesis. Auburn Univ.

ORTavant R., Mauléon P., Thibault C., 1964. Photoperiodic control of gonadal and hypophyseal activity in domestic mammals. Ann. N. Y. Acad. Sci ., 117, I 57-192.

Palmer R., I964. Celioscopic study of women treated with cyclic norethisterone for endometriosis. Int. J. Ferl., 9, I $21-122$.

Pelletier J., 1963. Étude critique du dosage de ICSH par la méthode de l'acide ascorbique ovarien. Ann. Biol. anim. Bioch. Biophys., 3, 307-323.

Preletier J., ORTAVANT R., I965. Variations saisonnières des activités hypophysaires et sexuelles chez le bélier Ile-de-France. XXIII Internat. Congr. Physiol. Sci., Tokyo, September I965 (Abst.).

REARdon T. F., Robinson T. J., I96r. Seasonal variation in the reactivity to œstrogen of the ovariectomized ewe. Aust. J. Agric. Res., 12, 320-326.

REEVES J. J., 1967. Pituitary gonadotropic activity of the ewe during the breeding and anestrous seasons. J. Anim. Sci., 26, 9.50. (Abst.)

Robertion H. A., Hutchinson J. S. M., 1962. The levels of FSH and LH in the pituitary of the ewe in relation to follicular growth and ovulation. J. Endocrin., 24, I43-15I.

Annales de Biologie animale. - 1969. 
Robinson T. J., 1959. The estrous cycle of the Ewe and Doe. In CoLE H. H., Cupps P. T. Reproduction in Domestic Animals, 1, 291-333. Academic Press, New York and London.

Roux L. L., 1936. Sex physiology of the sheep. Onderstepoort. J. Vet. Sci., 6, 465-714.

Smith I. D., I966. Estrous activity in Merino ewes in Western Queensland. Proc. Aust. Soc. Anim. Prod., 6, 69-79.

Steelman S. L., Pohley F. P., I953. Assay of follicle stimulating hormone based on the augmentation with hormone chorionic gonadotropin. Endocrinology, 53, 604-616.

Thibault C., Courot M., Martinet L., Mauléon P., du Mesnil du Buisson F., Ortavant R.,

Pelletier J., Signoret J. P., ig66. Regulation of breeding season and estrous cycles by light and external stimuli in some mammals. J. Anim. Sci., 25, I 19-142.

ThWAItEs C. J., 1965. Photoperiodic control of breeding activity in the Soulhdown ewe with particular reference to the effects of an equatorial light regime. J. Agric. Sci., 65, 57-64.

WARWICK E. G., 1946. Gonadotrophic potency of ewe pituiraty glands as affected by spaying, season and breed. Proc. Soc. Exp. Biol., 63, 530-533.

WATSON R. H., RADFORD H. M., 1960. The influence of rams on onset of cestrus in Merino ewes in the spring. Austr.. J. Agric. Res., 11, 65-7I.

Williams S. M., Garricus U. S., Norton H. W., Nalbandov A. V., 1956. Variations in the length of æestrus cycles and the breeding season in ewes. J. Anim. Sci., 15, 985-989.

YEates N.T. M., 1949. The breeding season of the ewe with particular reference to its modification by artificial means using light. J. Agric. Sci, 39, 1-43. 